\title{
El cuidado de la vida durante COVID-19, una mirada desde la investigación en salud
}

\author{
Caring for Life During COVID-19, Looking from Health Research
}

Cuidando da vida durante COVID-19, um olhar da pesquisa em saúde

Luis Fernando Marin Cadavid ${ }^{1}$

\section{Resumen:}

El objetivo es analizar el aporte de enfermería en la investigación en salud, para el cuidado de la vida en el contexto de la pandemia por COVID 19. Se presenta una recopilación, acerca de la importancia de la investigación en salud para el cuidado de la vida y cómo la enfermería hace aportes a este tema tan crucial para la humanidad durante la pandemia por COVID-19. Este escrito está dirigido a los estudiantes de las ciencias de la salud y toda persona que este incursionando en la investigación en salud, busca motivar la reflexión de aspectos tales como: de dónde surge la investigación, cómo se integra el cuidado y el proceso salud enfermedad de las comunidades, por qué es importante la investigación en salud, cómo la investigación en salud en general y de enfermería en concreto hacen aportes durante la pandemia por COVID-19 y cómo la disciplina hace su aporte a la investigación en salud, para poder ejercer su misión de cuidado de la vida.

\section{Palabras clave:}

Investigación en Enfermería, Atención en Enfermería, Infecciones por Coronavirus.

\section{Abstract:}

The objective is to analyze the contribution of nursing in health research, for life care in the context of the COVID 19 pandemic. A compilation is presented about the importance of health research for life care and how nursing contributes to this crucial issue for humanity during the COVID-19 pandemic. This writing is aimed at students of health sciences and anyone who is venturing into health research, seeks to motivate reflection on aspects such as: where does the research come from, how care and the health-disease process are integrated communities, why health research is important, how health research in general and nursing in particular make contributions during the

\footnotetext{
${ }^{1}$ Enfermero, estudiante de maestría en enfermería Universidad de Antioquia, Enfermero de emergencias en Clínica Las Américas AUNA. ORCID: 0000-0003-3920-4310.

e-mail:1fm526@gmail.com, luis.marin2@udea.edu.com
} 
COVID-19 pandemic and how the discipline makes its contribution to health research, in order to exercise his mission of caring for life.

\section{Keywords:}

Nursing Research, Nursing Care, Coronavirus Infections.

\section{Resumo:}

O objetivo é analisar a contribuição da enfermagem na pesquisa em saúde para o cuidado da vida no contexto da pandemia COVID 19. É apresentada uma compilação sobre a importância da pesquisa em saúde para o cuidado da vida e como a enfermagem contribui para essa questão crucial para a humanidade durante o COVID 19 pandemia. Esta redação é dirigida a estudantes de ciências da saúde e a todos que se aventuram na pesquisa em saúde, busca motivar a reflexão sobre aspectos como: de onde vem a pesquisa, como o cuidado e o processo saúde-doença são comunidades integradas, por que a pesquisa em saúde É importante, como a pesquisa em saúde em geral e a enfermagem em particular contribuem durante a pandemia COVID-19 e como a disciplina dá sua contribuição para a pesquisa em saúde, a fim de exercer sua missão de cuidar da vida.

\section{Palavras-chave:}

Pesquisa em Enfermagem, Cuidados de Enfermagem, Infecções por Coronavirus.

\section{Introducción}

El ser humano siempre se ha preocupado por darle explicación a los diferentes fenómenos que lo rodean, la búsqueda de respuestas le ha permitido desarrollar diversos procesos para generar conocimiento y dar sentido a su cotidianidad, y por qué no, para plantear nuevas inquietudes. La investigación es un camino en este ir y venir, por lo tanto, la actividad de investigar está orientada a dar respuesta a necesidades humanas, a despejar interrogantes, pretende solucionar problemas y dar explicación a fenómenos en todos los ámbitos: económico, social, ambiental, cultural, entre otros.
Investigar exige, en lo ético y lo práctico, la aplicación de un método que dependerá del tema y la forma en que se describe o comprende el fenómeno, esto con miras a darle una mayor veracidad a la información e interpretaciones obtenidas. Es por esto por lo que se ha desarrollado la investigación en diferentes campos del saber, siempre con miras a que esas singularidades sean tomadas en cuenta. Estos métodos de investigación han surgido de las propuestas que los diferentes científicos han realizado en cada momento de la historia que buscan que se ajusten mejor a las particularidades del estudio. 
Por otro lado, se han desarrollados diferentes campos de la investigación en todas las áreas del conocimiento, como la investigación educativa, la investigación cultural, la investigación social, la investigación detectivesca policial y la investigación en salud, por mencionar algunos de estos.

La investigación en salud contempla diferentes sub temas que agrupan fenómenos que dan respuesta a las dificultades que enfrenta el ser humano; su crecimiento obligó a convertirse en un campo científico y los diferentes aportes que le han realizado todas las disciplinas de la salud exigió que esta se fuera convirtiendo en un campo multidisciplinar en el que tiene mucha importancia la enfermería.

En este sentido, las disciplinas que la componen se comprometen con el cuidado de la vida como eje central de la salud y se preocupan por dar respuesta a las situaciones que van surgiendo y en este momento la afectación al ser humano que ha traído el COVID-19. Por lo tanto, enfermería esta llamada a brindar aportes desde la investigación que le de sustento a su ser disciplinar de cuidado de la vida.

Se presenta una reflexión acerca de la relación entre la investigación en salud, el cuidado de la vida y el aporte de enfermería en ambas.

La investigación y la importancia de la investigación en salud

La investigación en una sociedad tiene grandes ventajas, pues puede generar un cambio de la situación existente, por medio de la creación y divulgación del conocimiento pertinente para ese momento sociohistórico; puede apuntar a la solución de problemas que aquejan una comunidad, como afirma Robles "se investiga para transformar la realidad y con ello contribuir al mejoramiento de la calidad de vida en los seres humanos" (1). Pero no solo es necesario quedarse en la creación de nuevos conocimientos, también es importante que esos conocimientos sean compartidos y puestos en práctica en la sociedad. Igualmente, es imperativo que se posibilite una investigación que apunte también a dar solución a problemas comunes en el mundo globalizado, en este sentido, autores como Serra reafirman esa posición "Es prioridad incrementar el diseño de estudios que desde lo local mejoren lo nacional e inclusive pueda colocarnos en lo internacional" (2).

Los diferentes aportes que hace la investigación en una sociedad también pueden ser de ámbito económico y cultural, a lo cual Wasserman dice "la investigación genera riqueza y bienestar, culturas de innovación y competitividad, capacidad para apropiar tecnologías trasferidas de otros lugares, y establece una sólida capacidad para la negociación de recursos e ideas." ${ }^{(3)}$ También ayuda a la toma de decisiones gubernamentales de forma más asertiva y consciente, por lo que el mismo autor afirma "la necesidad de procesos de toma de decisiones basados en la evidencia y en la racionalidad y de la defensa de la una sociedad abierta y democrática ante tendencias autoritarias." (3).

La investigación tiene una retribución social muy grande, la cual se refleja en los avances que se ha tenido en el sector salud, ya que este ha impactado en las vidas de cada persona disminuyendo sus complicaciones por diferentes patologías y aumentando su expectativa de vida, ante esto Wasserman defiende esta posición "Seguramente uno de los campos más dinámicos de investigación en el mundo es el campo de la salud. Es también un campo donde se ha producido avances que afectan a cada persona, disminuyendo sufrimientos y prácticamente 
duplicando, en apenas un siglo, expectativa de vida de gran parte de la población humana del mundo" (3).

La investigación en salud tiene como objetivo crear conocimiento nuevo o algún tipo de evidencia documental que permita contribuir a dar respuesta a necesidades que tiene la población, el equipo de salud o las instituciones prestadoras de servicios de salud frente a la situación de salud que enfrenta sus comunidades ${ }^{(4)}$.

Por lo tanto, es importante, mejorar la calidad de vida de las personas y prolongar sus años de vida productivos; también puede ayudar a mejorar las fortalezas de la comunidad aumentando su capacidad productiva siendo una herramienta transformadora de entornos sociales, aspecto que coincide con lo planteado por Aguirre "La investigación en salud, puede entonces ser visualizada como la herramienta generadora de recursos humanos, instrumentos, metodologías, herramientas y servicios que permiten identificar, transformar y ampliar las fortalezas de la región; esto bajo una perspectiva de aprovechamiento y generación de recursos financieros, orientados al establecimiento de una sociedad basada en el conocimiento" (4).

El científico en este campo está obligado constantemente a estar interrogándose cada uno de los aspectos que se plantea, debido a que las condiciones de salud en el ser humano son absolutamente dinámicas, adicionalmente están influenciadas por diferentes condicionantes internos y externos, los cuales apuntan a requerir soluciones pertinentes para el momento sociohistórico en el cual se encuentra la comunidad.

La investigación en salud sirve a los pueblos y sus gobernantes para tomar decisiones asertivas y racionales, ayuda a optimizar recursos en la formulación de políticas, pues permite tener una visión de los problemas más incluyente, donde las soluciones de las problemáticas parten de las necesidades de la población, en este sentido, Aguirre considera "En este sentido, uno de los retos más grandes que enfrenta la investigación en salud, es la incorporación de los resultados e insumos intelectuales a la toma de decisiones sustentada. De igual manera, se debe fortalecer el diseño, ejecución y evaluación de políticas públicas en salud bajo una óptica incluyente, multidisciplinaria y metodológicamente sustentada." ${ }^{(4)}$.

Los gobernantes deberán apoyar la investigación en salud, para que esta cumpla eficazmente su función social, este apoyo puede partir desde la financiación de investigaciones, hasta la apertura de sus bases de datos, donde se pueda extraer información relevante para sintetizarla y analizarla, de manera que sea útil para la toma de decisiones y formulación de políticas públicas, Mejía plantea cómo esta experiencia está siendo revertida en la nación Argentina "La Secretaría de Gobierno de Salud (SGS), a través de sus direcciones, programas, institutos y organismos descentralizados, recopila grandes cantidades de datos de salud que resultan de suma utilidad para la definición de políticas públicas." (5).

Si se considera la diada salud enfermedad como proceso integral y dinámico de la vida, que puede sufrir alteraciones o afectaciones por diferentes factores, tales como sociales, psicológicos, medioambientales o biológicos; este tipo de alteraciones cobran relevancia social y científica; por lo tanto es indispensable conocer los modos más adecuados de prevenirlas, enfrentarlas o superarlas de forma, en que el ser humano pueda alcanzar una vida individual, social y económica fructífera, para lograr el 
cuidado de la salud, ya que este representa un recurso del diario vivir de una persona, quien a su vez aporta en su sistema de vida cotidiana y en si a todo lo que lo rodea ${ }^{(4)}$. En este sentido se han desarrollado instrumentos que identifican las brechas de los sistemas de salud y permiten identificar las políticas públicas, uno de estos es la Investigación en Políticas y Sistemas de Salud (IPSS), los resultados que arroje esta herramienta pueden redundar en decisiones que modifiquen los servicios en el sistema de salud ${ }^{(6)}$. A pesar de estos instrumentos siguen existiendo brechas en la utilización del conocimiento en el desarrollo de políticas de salud.

La detección de problemas de salud que aquejan a las comunidades también debe partir de los profesionales de la salud, Labbe considera "el "médico investigador" es un agente idóneo para detectar aquellos problemas de salud que necesiten ser resueltos y estudiarlos." (7), este llamado no es solo para la disciplina de la medicina , la invitación para detectar y abordar los principales problemas de la salud deben tener un abordaje multidisciplinarios, debido a que cada disciplina de la salud puede hacer un aporte muy importante desde su ontología, haciendo así investigaciones desde su visión disciplinar, para Aguirre "es la formación de equipos de salud multidisciplinarios, para incorporarlos al contexto de la investigación sistemática de los principales problemas de salud." ${ }^{(4)}$.

\section{Investigación en salud y la enfermería}

La salud es un proceso transversal a la misma vida, para conservarla o recuperarla se han desarrollado actividades como la prevención, diagnóstico, tratamiento y rehabilitación; estas actividades demandan que todos los actores del sistema generen estrategias que puedan garantizar seguridad, calidad, disponibilidad y acceso a los diferentes servicios de salud que se ofrecen en el sistema; estrategias y políticas que deberán ser abordadas desde la evidencia documental que requiere partir de las necesidades de las personas; en este sentido la investigación en salud es clave, puesto que ella busca generar el conocimiento que apunta al cuidado de la salud y de la vida ligando las necesidades de la comunidad en los resultados de las investigaciones en salud realizadas.

En ese orden de ideas, la enfermería hace un aporte fundamental en la investigación en salud, ya que tiene una perspectiva del cuidado de la vida que ha ido construyendo el desarrollo de la investigación en enfermería. Fue en la década de 1950, cuando empezó a plantarse la necesidad de fundar sus propias bases teóricas que le generaran una práctica profesional más racional; también se empezó a pensar en la necesidad de reflexionar su quehacer diario que apuntara a la construcción de conceptos propios de su profesión, esta reflexión generó que se iniciará en la década de los 60 la formación posgradual de enfermería, este desarrollo permitió la existencia de enfermeras preparadas en investigación, lo cual ayudó a la construcción del nuevo conocimiento propio en el campo de cuidado y esta a su vez condujo al desarrollo diciplinar de la enfermería ${ }^{(8)}$.

En los últimos 70 años, gracias a la formación posgradual en la que se han formado las enfermeras de todo el mundo, adquiriendo así un cuerpo de conocimiento alrededor del cuidado que le ha generado una base para su desarrollos disciplinar, como lo afirma Luengo "Enfermería, gracias a una producción ascendente de conocimientos, ha evolucionado como disciplina, 
lo que le ha permitido estructurar un cuerpo de conocimientos propios, sin cuya existencia el desarrollo disciplinar se vería estancado." (9) Rebolledo apoya esta idea atribuyendo ese desarrollo científico y disciplinar a la formación universitaria "En la actualidad, los cambios en enfermería se han hecho evidentes, sobre todo con la adquisición del nivel universitario y con las publicaciones científicas de la disciplina." (10). Estos desarrollos disciplinares han generado un enriquecimiento social a nivel mundial, en donde la enfermera tiene la posibilidad de ejercer cuidado partiendo de su propio desarrollo conceptual que ha construido a partir de las necesidades de cuidado, al respecto Valenzuela dice "El reconocimiento de la enfermería como una disciplina profesional implica que quienes la ejercen puedan establecer una relación directa entre el cuidado de enfermería, el desarrollo conceptual y el método seleccionado para proporcionar el cuidado" (11), también este avance en el cuerpo de conocimientos ha contribuido al cuidado de las personas desde diferentes perspectivas que mejoran no solamente a los individuos sino a las comunidades, como dice Eugenia "Otras teoristas definieron enfermería como un sustantivo o verbo que significaba "saber", considerando que la práctica de enfermería no es enfermería, sino más bien es el uso del conocimiento de enfermería para una mejoría de los seres humanos". ${ }^{(8)}$.

La enfermería se constituyó entonces en una profesión liberal y una disciplina profesional práctica que tiene como fin el cuidado de la salud del ser humano ${ }^{(12)}$ y su trabajo apunta al cuidado de la vida, esto le ha permitido abordar el fenómeno del cuidado y que el conocimiento que va a generar parta de su práctica profesional para desarrollar teoría y posteriormente volver a la práctica cotidiana de una forma mejorada (13) (14). Es así como la investigación de enfermería está al servicio del cuidado de la vida, ya que este es el fin último de la disciplina.

La investigación en enfermería se apoya en diferentes métodos para poder desarrollar el conocimiento propio disciplinar un ejemplo de esto es el uso de la investigación cualitativa que desde hace varias décadas viene influenciando varias disciplinas, esta tiene como objeto de estudio la comprensión de los fenómenos y las interacciones que se presenta entre las personas y en el caso de la disciplina de enfermería, la relación que se establece entre enfermera y/o profesional de la salud con sus pacientes, familia y comunidad. ${ }^{(15)}$.

El desarrollo investigativo que ha tenido la enfermería en estos últimos 70 años ha permitido proponer diferentes mejoras en la construcción de política públicas en salud en muchos lugares del mundo, en este sentido, el parlamento británico plantea en su informe titulado "The triple Impact" en el cual uno de sus puntos es realizar inversión en la que se potencie enfermería, específicamente la investigación que beneficie el cuidado de la vida. ${ }^{(16)}$ por lo anterior organizaciones como el Consejo Internacional de Enfermería (CIE), La Organización Mundial de la Salud (OMS), la Organización Panamericana de la Salud (OPS) declararon el año 2020 como el año internacional de la enfermeras y matronas que busca visibilizar la importancia y necesidad de las enfermeras en la población mundial, principalmente en la participación de estas en los organismos de toma de decisiones y en su contribución para el cumplimiento de los objetivos de sostenibilidad del Banco Mundial. ${ }^{(17)}$. 


\section{La investigación en enfermería y}

el COVID-19
El año 2020 la humanidad enfrenta la aparición de la infección por SARS- CoV-2 dando origen al síndrome de respiración aguda grave más conocido como COVID-19, se ha demostrado a la sociedad en general en diferentes medios de comunicación, la importancia de la profesión de enfermería, puesto que en esta pandemia ha estado en primera línea en la atención hospitalaria de los pacientes enfermos de COVID-19, también aportando con investigaciones y desarrollo disciplinar para hacer frente y control a la epidemia ${ }^{(18)}$; es de suma importancia, el desarrollo científico que tienen las profesiones de la salud y especial la profesión de enfermería, pues es esta la que hace un cuidado y acompañamiento directo a las personas que están enfrentando complicaciones por el COVID-19.

Por lo tanto, la investigación de enfermería le hace un aporte importante al cuidado de la salud, y a la vez le ayuda al ser humano a estar mejor en todas sus dimensiones, como afirma Valenzuela "el "cuidado" de origen ancestral, esencia del quehacer de nuestra disciplina, tiene una mirada diferente: ve al sujeto como enfermo-sujeto antes que su enfermedad-objeto, lo cual permite que el sufrimiento que puede ser aliviado no sea "ocultado" en la profundidad biológica del organismo." ${ }^{(11)}$. Al estar presente en cuidado en todas las dimensiones del ser humano, la enfermería no iba a estar ajena a la crisis causada por la pandemia mundial que enfrenta la humanidad por el COVID-19, este profesional ha estado en primera línea en la atención de los pacientes, tanto en el ámbito hospitalario como extrahospitalario; ha demos- trado su importancia en el cuidado de la vida, como afirma fuentes "Aun cuando el hospital y particularmente las unidades de cuidado intensivo han sido lugares de especial importancia durante la crisis, otros ámbitos de cuidado deben valorarse por su capacidad para mitigar el sufrimiento humano." ${ }^{(18)}$ enfermería ha mostrado su importancia estando presente como profesión en el acompañamiento en hogares geriátricos o en entidades gubernamentales donde se brinda un cuidado a la población en tiempos de pandemia, más allá de lo hospitalario, de esta manera a la vez que ofrece cuidado, además, ha mostrado con trabajos de investigación la problemática y el avance en los cuidados ${ }^{(19)(20)}$.

\section{Conclusiones}

Es difícil pensar en investigación que vaya en contravía a cuidar o mejorar la calidad de vida de los seres humanos, esto ha sido muchas veces analizado en la historia, de tal manera que se han sacado declaraciones internacionales, acuerdos académicos, legislaciones en cada uno de los países y pronunciamientos al respecto las diferentes agremiaciones profesionales, pues en general, lo que se busca siempre es sopesar el daño que se va generar con la investigación versus el ganancia que van a tener los seres humanos o comunidades de personas al realizar esta. La investigación en salud siempre va a apuntar al cuidado de la vida mejorando la forma cómo se vive en cada momento sociohistórico, las visiones y las formas de abordar los fenómenos de salud van a variar dependiendo la disciplina que 
lo haga, pero indistintamente cual es el grupo profesional que realice la investigación en salud, el conocimiento debe apuntar al cuidado de la vida y mejorar el bienestar del ser humano.

Todas las disciplinas de la salud en tiempos de pandemia por el SARS- CoV-2 han contribuido en la búsqueda de soluciones que minimicen el impacto desbastador que puede tener esta epidemia en la humanidad, y cómo poder enfrentarla y superarla de la mejor forma más adecuada; demostrando así la importancia y necesidad de aumentar la inversión en investigación en el campo de la salud.

Las enfermeras son una piedra angular en los sistemas de salud y esto ha sido ampliamente demostrado en medio de la pandemia por SARSCoV-2 donde el desarrollo disciplinar e investigativo, ha hecho que esta profesión muestre su alto compromiso con el cuidado de la salud y de la vida humana.

La enfermería hace un aporte al cuidado de la vida, ya que este es su campo de desarrollo disciplinar, por ende, todas las investigaciones que se realicen en esta disciplina van a ir encaminadas al mejoramiento de la práctica profesional, cuyo objetivo en ultimas es el cuidado de la vida.
1. Robles Mirabal V, Serrano Díaz CA, Barrios Rodríguez $\mathrm{T}$, González Ortega DM. La investigación científica como vía de superación profesional. EDUMECENTRO [Internet] 2019; 11(2): 220-4. Disponible en: http://scielo.sld. $\mathrm{cu} /$ scielo.php? script $=$ sci_arttext $\&$ pi $\mathrm{d}=$ S2077-28742019000200220\&lng=es [consulta: 11 abr 2020].

2. Serra Valdes MA. La investigación en la formación de profesionales y en el profesorado de las ciencias médicas. Educación Médica Superior [Internet] 2018 31(4). Disponible en: http://www.ems.sld.cu/index.php/ems/article/view/1194 [consulta: 11 abr. 2020].

3. Wasserman M, Sobre la importancia de investigar en Colombia, un país subdesarrollado. Biomédica [Internet] 2001; 21(1):1324. Disponible en: https://www.redalyc. org/articulo.oa?id=84321103 [citado 11 abr. 2020].

4. Aguirre Crespo A. Panorama de la investigación en las ciencias de la salud. Rev Salud Quintana Roo [Internet] 2018; 11(39): 5-6. Disponible en: https://www.medigraphic.com/pdfs/salquintanaroo/sqr-2018/ sqr1839a.pdf [consulta: 11 abr. 2020].

5. Mejía R, Palmero A. La Secretaría de Gobierno de Salud de la Nación regula el acceso a los datos de salud con fines de investigación. Rev Argent Salud Pública [Internet] 2019; 10(40): 5-6. Disponible en: http://rasp.msal.gov.ar/rasp/articulos/volumen40/5-6.pdf [consulta: 11 abr. 2020]. 
6. Martínez-Trujillo N, Corrales-Fernández N, Peña-Figueredo MA. Capacidades para la gestión de resultados de investigación en sistemas y servicios de salud de enfermería en Cuba. Rev enferm Inst Mex Seguro Soc [Internet] 2019; 27(2): 116-21. Disponible en: https://www.medigraphic.com/pdfs/ enfermeriaimss/eim-2019/eim192h.pdf [consulta: 18 jul. 2020].

7. Labbé TP, Barake MF, Álamos MF, Molina CG, Ríos JA. Realidad de la investigación en salud en Chile: participación de los médicos en el Fondo Nacional de Investigación (FONIS) en la última década. ARS Médica [Internet] 2017; 42(2): 76-80. Disponible en: https://arsmedica.cl/index. php/MED/article/view/933/892 [consulta: 26 jul. 2020].

8. Urra M E. Avances de la ciencia de enfermería y su relación con la disciplina. Cienc enferm [Internet] 2009;15(2): 9-18. Disponible en: https://scielo.conicyt.cl/scielo.php?script $=$ sci_arttext $\&$ pi$\mathrm{d}=$ S0717-95532009000200002\&lng=es [consulta: 13 abr. 2020].

9. Luengo-Martínez CE, Sanhueza-Alvarado O. Formación del licenciado en Enfermería en América Latina. Aquichan [Internet] 2016; 16(2): 240-55. Disponible en: http://www.scielo.org. co/scielo.php?script $=$ sci_arttext \&pi $\mathrm{d}=$ S1657-59972016000200011\&lng=en [consulta: 11 abr. 2020].

10. Rebolledo-Malpica D. Enfermería según la perspectiva deconstructivista de Derrida. Rev Fac Med [Internet] 2019; 67(1): 91-6. Disponible en: http://www.scielo. org.co/scielo.php?script=sci_arttext\&pi$\mathrm{d}=$ S0120-00112019000100091 [consulta: 18 jul. 2020].
11. Valenzuela-Suazo SV. Aplicación de la teoría en la investigación del cuidado. Rev enferm Inst Mex Seguro Soc [Internet] 2019; 27(3): 124-7. Disponible en: http:// revistaenfermeria.imss.gob.mx/editorial/ index.php/revista_enfermeria/article/ view/1101/1038 [consulta: 24 jul. 2020].

12. Se reglamenta la profesión de enfermería en Colombia y se dictan otras disposiciones. Ley 266 de 25 de enero de 1996. [Internet]. Colombia. Disponible en: https:// www.anec.org.co/images/Documentos_ ANEC/ley_266_1996.pdf [consulta: 11 abr. 2020].

13. Gálvez Díaz N, Gonzáles Hernández Y, Monsalve Menor M. Actitud hacia la investigación científica al final de la carrera de Enfermería en Perú. Gac Med Bol [Internet]. 2019; 42(1): 32-7. Disponible en: http://www.scielo.org. bo/scielo.php?script=sci_arttext\&pi$\mathrm{d}=\mathrm{S} 1012-29662019000100006 \& \operatorname{lng}=\mathrm{es}$ [consulta. 26 jul. 2020].

14. Alberto L. Formación para la ciencia: una reflexión sobre los desafíos de la investigación en enfermería en la región de Latinoamérica. Rev Cuid [Internet] 2014; 5(2): 713-6. Disponible en: https://revistas.udes.edu.co/cuidarte/article/view/128 [consulta: 26 jul. 2020].

15. Juanillo-Maluenda H. Posicionamiento del investigador de enfermería en la utilización de la teoría fundamentada constructivista. Enfermería Univ [Internet] 2019; 16(2): 205-15. Disponible en: http://www.scielo. org.mx/scielo.php?script=sci_abstract\&pi$\mathrm{d}=$ S1665-70632019000200205\&lng=es\&n$\mathrm{rm}=$ iso [consulta: 18 jul 2020]. 
16. Domenech-Climent N. 2020, el año de la enfermera. Enfermería Oncológica [Internet] 2020; 22(1): 56-60. Disponible en: https://revista.proeditio.com/enfermeriaoncologica/article/view/2203 [consulta: 02 set. 2020].

17. Velásquez Jiménez CM. 2020, el año de la enfermería en Colombia y sus retos ante la pandemia por COVID-19. Rev Colomb Enferm [Internet] 2020; 19(2): e019. Disponible en: https://revistacolombianadeenfermeria.unbosque.edu.co/index.php/ RCE/article/view/e019/2784 [consulta: 02 set. 2020].

18. Fuentes-Bermúdez GP. Enfermería y COVID-19: reconocimiento de la profesión en tiempos de adversidad. Rev Colomb Enferm [Internet] 2020; 19(1): e017. Disponible en: https://revistacolombianadeenfermeria.unbosque.edu.co/index.php/ RCE/article/view/2970/2331 [consulta: 02 set. 2020].
19. Canova-Barrios C, Salvador S. Proceso Enfermero en la infección por COVID-19: y entonces, ¿Cómo procedemos? Cuidados de Enfermería y Educación en Salud [Internet] 2020; 5(1): 37-51. Disponible en: https://www.researchgate.net/publication/344501878_Proceso_Enfermero_en_ la_infeccion_por_COVID-19_y_entonces_ Como_procedemos [consulta: 02 set 2020]. 20. Acevedo Gamboa FE, Páez Barrozo M, Mayorga Camargo VL. Enfermería de urgencias en la intubación de secuencia rápida a pacientes COVID-19. Revista Cuidarte [Internet] 2020; 11(3): e1319. Disponible en: https://revistas.udes.edu.co/cuidarte/ article/view/1319/1745 [consulta: 23 set 2020].

Recibido 02.10.20

Aceptado 29.05.21 